Contexto educativo: convergencias y retos desde la perspectiva psicológica, es una obra que pretende generar un espacio de discusión entre diferentes campos de la psicología educativa y el rol del psicólogo; se tiene como objetivo promover una mirada integral de los contextos escolares y los nuevos retos para un país más educado. Para ello, se realizó un análisis desde los diferentes momentos del desarrollo escolar, del educando y la práctica del docente. Así mismo, se estudiaron las características de los contextos educativos con una mirada interdisciplinaria y transcultural. Los hallazgos y discusiones aquí presentadas, son el resultado de rigurosos estudios en el marco de la psicología y la educación, proyectos que tributan a grupos de investigación de prestigiosas instituciones, reuniendo autores de países latinoamericanos como Ecuador, Perú y Venezuela; así como profesionales colombianos de ciudades como Cali, Bogotá, Bucaramanga, Barranquilla y Pamplona. Este libro se convierte en obra fundamental de consulta para los apasionados por la psicología educativa, psicólogos en formación, educadores e investigadores, debido a su mirada integradora y a su perspectiva de futuro en busca de una mejor sociedad para todos.

\section{CONTEXTO EDUCATIVO:}

CONVERGENCIAS Y RETOS

DESDE LA PERSPECTIVA PSICOLÓGICA

Compiladores:

Manuel Ernesto Riaño Garzón - Sandra Milena Carrillo Sierra Javier Leonardo Torrado Rodríguez - Jhon Franklin Espinosa Castro 


\title{
CONTEXTO EDUCATIVO: CONVERGENCIAS Y RETOS DESDE LA PERSPECTIVA PSICOLÓGICA
}

\author{
Compiladores: \\ Manuel Ernesto Riaño Garzón \\ Sandra Milena Carrillo Sierra \\ Javier Leonardo Torrado Rodríguez \\ Jhon Franklin Espinosa Castro
}

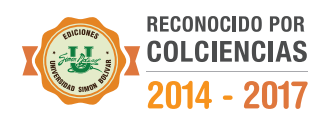




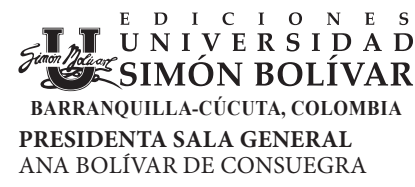

RECTOR FUNDADOR

JOSÉ CONSUEGRA HIGGINS (Q.E.P.D.)

RECTOR EJECUTIVO

JOSÉ CONSUEGRA BOLÍVAR

VICERRECTORA ACADÉMICA

SONIA FALLA BARRANTES

VICERRECTORA DE INVESTIGACIÓN E INNOVACIÓN

PAOLA AMAR SEPÚLVEDA

VICERRECTORA FINANCIERA

ANA CONSUEGRA DE BAYUELO

SECRETARIA GENERAL

ROSARIO GARCÍA GONZÁLEZ

DIRECTOR GENERAL SEDE CÚCUTA

TOMÁS WILCHES BONILLA

DIRECTORA ACADÉMICA, DE INVESTIGACIÓN

E INNOVACIÓN SEDE CÚCUTA

SANDRA WILCHES DURÁN

DIRECTORA ADMINISTRATIVA, FINANCIERA

Y DE INFRAESTRUCTURA SEDE CÚCUTA

MYRIAM WILCHES DURÁN

DIRECTOR JURÍDICO SEDE CÚCUTA

WILLIAM TOMÁS WILCHES DURÁN

COORDINADOR DE INVESTIGACIONES

SEDE CÚCUTA

JULIO CÉSAR CONTRERAS VELÁSQUEZ

DEPARTAMENTO DE PUBLICACIONES

CARLOS FEDERICO MIRANDA MEDINA

MARÍA INÉS MENDOZA BERNAL

JHON FRANKLIN ESPINOSA CASTRO

KEVIN DE JESÚS VILLAREAL JIMÉNEZ

MIEMBROS DE LA SALA GENERAL

ANA BOLÍVAR DE CONSUEGRA

OSWALDO ANTONIO OLAVE AMAYA

MARTHA VIVIANA VIANA MARINO

JOSÉ EUSEBIO CONSUEGRA BOLÍVAR

JORGE REYNOLDS POMBO

ÁNGEL CARRACEDO ÁLVAREZ

ANTONIO CACUA PRADA

JAIME NIÑO DÍEZ

ANA CONSUEGRA DE BAYUELO

JUAN MANUEL RUISECO

CARLOS CORREDOR PEREIRA

JORGE EMILIO SIERRA MONTOYA

EZEQUIEL ANDER-EGG

JOSÉ IGNACIO CONSUEGRA MANZANO

EUGENIO BOLIIVAR ROMERO

ÁLVARO CASTRO SOCARRÁS

IGNACIO CONSUEGRA BOLÍVAR 


\title{
CONTEXTO EDUCATIVO: CONVERGENCIAS Y RETOS DESDE LA PERSPECTIVA PSICOLÓGICA
}

\author{
Compiladores: \\ Manuel Ernesto Riaño Garzón \\ Sandra Milena Carrillo Sierra \\ Javier Leonardo Torrado Rodríguez \\ Jhon Franklin Espinosa Castro
}

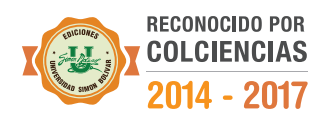




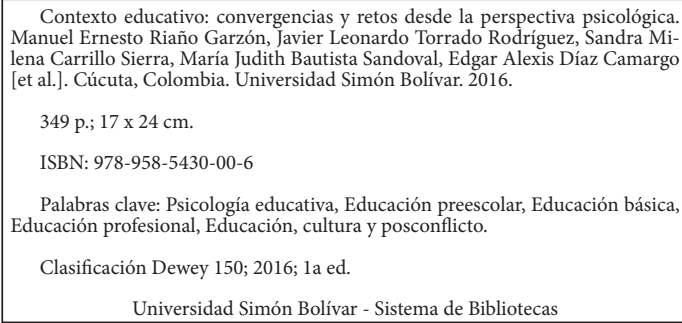

Universidad Simón Bolívar - Sistema de Bibliotecas

\title{
CONTEXTO EDUCATIVO: CONVERGENCIAS Y RETOS DESDE LA PERSPECTIVA PSICOLÓGICA
}

\section{Compiladores:}

@Manuel Ernesto Riaño Garzón, @Sandra Milena Carrillo Sierra, (C)avier Leonardo Torrado Rodríguez, OJhon Franklin Espinosa Castro

\author{
Autores: \\ ๑ Manuel Ernesto Riaño Garzón, @Javier Leonardo Torrado Rodríguez, @Sandra Milena Carrillo Sierra, \\ @ María Judith Bautista Sandoval, @Edgar Alexis Díaz Camargo, @Yanela Paola Jaimes Parada, \\ ๑) Oriana Marcela Chacón Lizarazo, (Ana María Arias Cardona, @Ana Carolina Rodríguez Ibarra, \\ ๑Marbel Lucía Gravini Donado, @Marinella Beatriz Álvarez Borrero, @Lilibeth Monterroza Ursprung \\ @S Sandra Licette Padilla Sarmiento, @Diana Janeth Villamizar Carrillo, @Óscar Gilberto Hernández, \\ ๑Álvaro Ramírez Botero, @Alejandro Oses Gil, @Sandra Lorena Roa Cordón, \\ @Sebastián Jiménez Jiménez, @Johana Cristina Palma López, @María Luisa Montánchez Torres, \\ ๑ José David Gil Montoya, @Mónica Julieth Jaimes Martinez, @Hugo David Salgado Zambrano, \\ ๑) Cindy Tatiana Parra Oquendo, @Rosario Gildemeister Flores, @Rosina Vanessa Sánchez Jiménez, \\ ๑) Patricia Vergara Bao, @Micaela Wetzell Espinoza, @Jovany Gómez Vahos, \\ ( )Kattia Magred Sepúlveda Rodríguez, @Jaime Iván Silva Calderón, @Nidia Johanna Bonilla Cruz.
}

ISBN: 978-958-5430-00-6

GRUPOS DE INVESTIGACIÓN:

Proyecto: Relación existente entre la regulación-control de conducta, prácticas de crianza y características sociodemográficas en niños que inician edad escolar.

DESARROLLO HUMANO, EDUCACIÓN Y PROCESOS SOCIALES

Proyecto: Caracterización de las funciones ejecutivas en estudiantes de grado 11, de las Instituciones Educativas Públicas del municipio de Cúcuta.

\section{ALTOS ESTUDIOS DE FRONTERA (ALEF)}

Todos los derechos reservados. Ninguna parte de esta publicación puede ser reproducida, almacenada en sistema recuperable o transmitida en ninguna forma por medios electrónico, mecánico, fotocopia, grabación u otros, sin la previa autorización por escrito de Ediciones Universidad Simón Bolívar y del autor. Los conceptos expresados de este documento son responsabilidad exclusiva de los autores y no necesariamente corresponden con los de la Universidad Simón Bolívar. Esta obra cumple con el Depósito Legal según lo establecido en la Ley 44 de 1993, los Decretos 460 del 16 de marzo de 1995, el 2150 de 1995, el 358 de 2000 y la Ley 1379 de 2010.

\section{(C)Ediciones Universidad Simón Bolívar}

Carrera 54 No. 59-102

http://publicaciones.unisimonbolivar.edu.co/edicionesUSB/

dptopublicaciones@unisimonbolivar.edu.co

publicacionescucuta@unisimonbolivar.edu.co

Barranquilla - Cúcuta

Marzo de 2017

Cúcuta

Printed and made in Colombia 


\title{
CApítulo 3 \\ Ia intervención psicoeducativa y la psicología educativa: Una diferencia necesaria*
}

\author{
Álvaro RAMÍrez Botero ${ }^{1}$
}

\section{Resumen}

Actualmente existen consideraciones sobre lo que es psicología educativa y en esa categoría se enmarcan una serie de intervenciones que se llevan a cabo desde los diferentes campos aplicados de la psicología, desde otras disciplinas o en diferentes ámbitos institucionales. El efecto de lo anterior ha discurrido entre el intento de cada ámbito por fortalecerse llevando a diferentes contextos sus aplicaciones y a las reflexiones y discusiones académicas y gremiales por los límites, alcances y cruces de la psicología en cada ámbito. Por esto desde las indagaciones derivadas del trabajo de tesis doctoral "La institucionalización de la psicología educativa en Colombia", se establecen algunas diferencias entre lo las intervenciones psicoeducativas y la psicología educativa.

Palabras clave: Intervención psicoeducativa, Psicología educativa, Psicología de la educación, Institucionalización, Institución.

\footnotetext{
Este trabajo hace parte de los avances de la tesis doctoral "La institucionalización de la psicología educativa en Colombia".

1 Psicólogo, U. de A. Magíster en Educación P.U.J. y U. de M. Candidato Doctor Humanidades, Universidad EAFIT. Decano Facultad Ciencias Sociales, Institución Universitaria de Envigado. Docente cátedra Programa de Psicología, Universidad EAFIT. alrabote@yahoo.es
} 


\begin{abstract}
Nowadays it exits some considerations about what educational psychology is, and in this category a series of interventions are framed, categories that carry through from different fields applied to psychology, from other disciplines or in different institutional milieus. The effect of the latter has gone within the intent of each environment to get stronger by bringing to different contexts its applications, and to the reflections and academic and professional discussions by means of boundaries, milestones, and exchanges of psychology in each environment. Therefore, from the inquiries derived from the product of the doctoral thesis "The institutionalization of the educational psychology in Colombia”, some differences are established between the psycho-educational interventions and the educational psychology.
\end{abstract}

Keywords: Psycho-educative intervention, Educational psychology, Psychology of education, Institutionalization, Institution.

\title{
Desarrollo de contenidos
}

\section{Planteamientos sobre la psicología educativa}

En la práctica psicológica y en la producción académica en psicología, se presentan confusiones entre las intervenciones psicoeducativas y la psicología educativa ${ }^{2}$. Estas confusiones pueden perfilar a la psicología educativa de manera similar a la que se ha perfilado la psicología clínica: como una serie de teorías, conceptos y técnicas que se pueden trasponer a cualquier ámbito de aplicación de la psicología desconociendo la posible especificidad que la puede hacer parcela de conocimiento y una profesión:

Creo que la especificidad de la psicología de la educación es más cuestionada por los colegas psicólogos que trabajan en otros ámbitos de la psicología y que tienden más bien a ver a la psicología de

2 Como se puede notar de entrada se propone que existe diferencia entre la psicología de la educación y las intervenciones psicoeducativas. En estos términos la discusión corresponde también a la diferenciación entre el uso de la psicología de la educación como ciencia básica y como ciencia aplicada. 
la educación como un "campo de aplicación" de un conocimiento científico psicológico generado por la psicología del aprendizaje, por la neuropsicología, por la psicología experimental, y que tienden a considerar a la psicología de la educación -como la [psicología] clínica o como la social- como una "contextualización" de estos conocimientos universales científicos que proporciona la investigación psicológica básica. (Rigo, Díaz \& Hernández, 2005, p.4)

Al respecto de dicha especificidad, Coll (1997), presenta la existencia del acuerdo sobre la psicología de la educación como

"la aplicación de los principios y explicaciones de la psicología a la teoría y las prácticas educativas" (p.15) y la del desacuerdo entre los especialistas sobre definir "en qué consiste esta aplicación, qué contenidos incluye, cómo integrar sus aportaciones en una aplicación global y no reduccionista de los procesos educativos, qué relaciones mantiene con las otras parcelas de la psicología científica, cuál es el perfil profesional del psicólogo de la educación, etc.” (p.15)

En el esfuerzo por aclarar los alcances y los límites de la psicología de la educación Coll la define como "uno de los ámbitos del conocimiento, de la actividad científica y de la actuación profesional que contribuye a la elaboración de una teoría educativa y a fundamentar una práctica pedagógica de acuerdo con ella" (1998, p.13), a esta definición se suma otra afirmación del mismo autor en la misma obra: "La psicología de la educación es uno de los pilares del ámbito de conocimiento que llamamos psicopedagogía" (p.17). Así se evidencia la intersección entre discursos y la comprensión de la salida propuesta por Coll (1997) con el planteamiento de la psicología de la educación como disciplina puente en tanto es "una disciplina psicológica y educativa de naturaleza aplicada” (p.16). Esta concepción ha tenido gran aceptación y ha permitido pensar el lugar de la psicología de la educación como saber específico y del psicólogo educativo como profesional. 
Sin embargo, no se puede pasar por alto que muchas veces al hablar de psicología de la educación automáticamente se piensa en la escuela y en la educación formal. Este efecto que también señala Coll (2004a, p.5), corresponde al ascenso de las instituciones modernas, entre las que está la escuela, a la que se le han trasladado responsabilidades de otras instituciones modernas que cumplen con prácticas sociales de control y de socialización ${ }^{3}$.

La importancia que reviste a la escuela como institución invita a considerar que sobre ella se afirma que es el "aparato ideológico dominante de la sociedad capitalista" (Althusser, 1976, p.46) y que junto con los otros aparatos ideológicos del Estado cumple la función de reproducir las relaciones de producción. Pero el orden social se reacomoda y hoy está alineado en lo que se denomina como la sociedad de la información. En el nuevo orden la escuela continúa con un papel importante y también sufre grandes transformaciones en su interior y la educación traspasa las dinámicas escolares: La idea de aprendizaje formal escolar de los dos últimos siglos se ve afectada con las posibilidades de aprendizaje no formal e informal (Coll, 2004b) y esto necesariamente implica a la psicología de la educación pues sus objetos de estudio y de intervención constituidos por las variables que definen los espacios educativos "Unos actores particulares con unos roles y unos formatos de interacción establecidos, unos contenidos concretos y unas modalidades de organización del tiempo, el espacios y los recursos específicos" (Coll \& Monereo, 2008, p.35) también se ven modificados por las TIC.

En este contexto la psicología de la educación mantiene su carácter de psicología aplicada y la emergencia de la psicología aplicada como tecnología social -ciencia aplicada- no la limita a ser una simple aplicación de la ciencia pura, sino que también le permite gozar de un carácter innovador (Coll, 1983) puesto que se adapta y se renueva en sus concepciones y

3 Es importante señalar que a este efecto no ha escapado la psicología que en sus diversos campos ha encontrado lugar de aplicación en dichas instituciones como la cárcel, el manicomio, los hospitales, la familia y por supuesto la escuela. 
técnicas de intervención. En esta línea se propone entender la psicología educativa como tecnología, y entender la tecnología como "un cuerpo de conocimientos prácticos" (Ardila, 2011, p.65) que se basa en la psicología como ciencia; mientras que la ciencia impacta la cultura y le interesa comprender, la tecnología impacta la sociedad y le interesa modificar (p.66); "la ciencia es una, y no existe ciencia aplicada sino ciencia por aplicar" (p.69). Algo similar sucede con la investigación: "La investigación aplicada" posee la mayoría de las características de la "investigación pura", sin embargo, su propósito es práctico: intenta mejorar un producto o proceso, generalmente probar concepciones teóricas en situaciones problema reales" (Rico, 1997, p.79).

En medio de las transformaciones sociales la afirmación de Coll (2004a, p.2) y Coll y Monereo (2008, p.20) de no circunscribir la psicología de la educación a la escuela, ha derivado en una salida para categorizar como psicología de la educación intervenciones en psicología de la salud, psicología organizacional, psicología deportiva, psicología social, psicología del consumidor y en otros ámbitos de la psicología, desconocen la naturaleza de la psicología de la educación y de los otros campos de la psicología, como ciencia aplicada con una identidad como saber específico (ciencia)y como rol profesional (profesión).

Alrededor de estas "aplicaciones" de la psicología de la educación, se potencia la discusión sobre si es una ciencia básica o una ciencia aplicada, puesto que funcionalmente su uso en diferentes ámbitos de la psicología, sin ubicar dichas acciones en el contexto institucional y en el orden del discurso que allí deviene deriva en aplicacionismo y queda sugerida como ciencia básica. Al respecto Coll (1983) apoyado en los desarrollos de Caparós y Bunge, se ocupó de establecer de manera clara por qué la psicología de la educación es una ciencia aplicada y no una ciencia básica: "la ciencia básica tiene como meta conocer el mundo mientras que la ciencia aplicada persigue el conocimiento del mundo para controlarlo" (p.2). Al hacerla 
funcionar como "ciencia básica la psicología educativa es desarraigada de su especificidad que se soporta en los orígenes en las situaciones educativas" (Castejón, González, Gilar \& Miñano, 2010, p.23). Mientras que usada como ciencia aplicada ${ }^{4}$, esta disciplina, puede mantener su especificidad estableciendo, por un lado interdependencia con los otros ámbitos de la psicología y, por otro, independencia de los mismos. Esta diferenciación se hace relevante cuando se observan las acciones de los psicólogos en ámbitos diferentes a la escuela: cuando las intervenciones psicoeducativas funcionan en una perspectiva aplicacionista de la psicología educativa -aunque sea en la escuela- se observarán ajenas, distantes, sin conexiones que den muestra de la interacción con el contexto institucional, lo mismo sucede cuando en la escuela hacen intervenciones propias de la psicología clínica: "Asimismo es claro que la misma institución escolar no es un contexto psicoterapéutico adecuado, razón por la cual, las dificultades de corte psicológico que demandan mayor atención que el apoyo u orientación emocional, deben tratarse en un entorno clínico propiamente dicho" (Hernández, 2012, p.37). En esta línea de discusión se presenta la siguiente cita de Foucault (2005) que opera como resorte en la discusión de la psicología educativa como ciencia aplicada y las intervenciones psicoeducativas como técnicas enmarcadas en contextos institucionales:

El deseo dice: "No querría tener que entrar yo mismo en este orden azaroso del discurso, no querría tener relación con cuanto hay en él de tajante y decisivo: querría que me rodeara como una transparencia apacible, profunda, indefinidamente abierta, en la que otros responderían a mi espera, y de la que brotarían las verdades, una a una: yo no tendría más que dejarme arrastrar, en él y por él, como algo abandonado, flotante y dichoso". Y la institución responde: "No hay

4 A partir de lo mencionado antes sobre la ciencia básica y la ciencia aplicada se establece la diferencia entre la psicología educativa como ciencia aplicada y el aplicacionismo de la psicología educativa. En la segunda se terminan usando sus desarrollos como si ella fuera una ciencia básica, esto es descontextualizados y esto solo aporta a la finalidad de comprender y teorizar en psicología. 
por qué tener miedo de empezar: todos estamos aquí para mostrarte que el discurso está en el orden de las leyes, que desde hace mucho tiempo se vela por su aparición: que se le ha preparado un lugar que le honra pero que le desarma, y que, si consigue algún poder, es de nosotros y únicamente de nosotros de quien lo obtiene". (Foucault, 2005, pp.12-13)

Se pretende, entonces, virar la discusión hacia el terreno de las instituciones para presentar algunos aportes funcionales para la diferenciación entre las intervenciones psicoeducativas y la psicología educativa desde los marcos institucionales y los discursos que las contienen y mostrar que el campo al que pertenecen las intervenciones psicoeducativas no es por defecto la psicología educativa sino que puede ser la psicología de la salud, la organizacional o la social entre otras, y esto lo define la circunscripción institucional y el discurso marco en el cual discurre la intervención.

\section{La institucionalización de las acciones sociales}

Entender la psicología educativa y las intervenciones psicoeducativas desde los marcos institucionales posibilita el fortalecimiento de la primera como saber específico y como profesión y de la segunda como una práctica de aplicación, que si no se instala en el orden discursivo de la institucionalidad que la contiene podrá quedar reducida a un aplicacionismo que arrastra a la psicología educativa al terreno de las aplicaciones del conocimiento universal básico de la psicología en los diferentes contextos bajo la consideración de que existen "leyes del comportamiento independientes del contexto" (Rigo, Díaz \& Hernández, 2005, p.5). La línea de diferenciación planteada es muy sutil, y a ella se suman los desacuerdos entre los psicólogos educativos:

No hay un acuerdo generalizado en torno a si la psicología de la educación tiene como objetivo principal la aplicación de conocimientos psicológicos relevantes al proceso educativo, centrando la inquietud 
investigadora en su vertiente aplicativa, o si ha de ser una ciencia aplicada que desarrolle sus propios programas de investigación y utilice técnicas y métodos adecuados de experimentación expresamente desarrollados para abordar los problemas educativos. (Beltrán \& Bueno 1995, p.14)

La psicología educativa tiene su origen en las instituciones educativas ${ }^{5}$, algo similar sucede a los otros ámbitos en la medida que los enmarca la institucionalidad para su existencia. Esto hace pertinente invitar a este escenario a algunos clásicos de la sociología y considerar que "las condiciones políticas y económicas de existencia no son un velo o un obstáculo para el sujeto de conocimiento sino aquello a través de lo cual se forman los sujetos de conocimiento" (Foucault, 1983, p.32). Reconocer algunos aspectos de estas relaciones contribuye al establecimiento de los lugares institucionales que contienen y definen las prácticas. Para el efecto se procede a identificar algunos elementos relevantes para este análisis en Marx, Parsons, Weber y Durkheim.

En primer lugar Marx (1959) expresa que los hombres representan papeles económicos (son actores): "en el transcurso de nuestra investigación hemos de ver que los papeles económicos representados por los hombres no son más que otras tantas personificaciones de las relaciones económicas en representación de las cuales se enfrentan los unos a los otros" (p.48). Las instituciones tienen gran relación con las fuerzas de producción, las relaciones de producción y las relaciones sociales -la división y la organización del trabajo- operan como la estructura de la sociedad donde se ubica el derecho que regula la división y la organización. Las instituciones funcionan como la superestructura que determina la estructura económica de la sociedad, funcionan como entidades generales, como ideas que se imponen

5 Si bien en la actualidad es prácticamente imposible pensar la escuela y la pedagogía sin la psicología y la psicología educativa como saber específico al margen de la pedagogía o de la escuela, las intervenciones psicoeducativas, como tecnologías derivadas de la psicología educativa, pueden pensarse en la escuela y por fuera de ella. 
al pensamiento y funcionan como "artículos de fe", algo en lo que se cree (Marx, 1978, pp.44-45).

Por su parte, Parsons (1961, p.41) plantea que la estructura social en su normativa cultural genera unas expectativas, estas tienen que ver con elementos del ambiente -social- que propone unos roles institucionalizados, preestablecidos. El mecanismo social de diferenciación de roles hace necesario establecer la diferencia entre valores y normas. Los valores son más universales, son lo deseable, generan expectativas pero difieren de los derechos y obligaciones; las normas son más específicas y están definidas dentro de un ámbito de significación más particular.

Max Weber (1997, p.5) propone que en la conjunción de las acciones sociales de varios individuos se establece la relación social, los participantes de ella orientan su acción hacia los otros en las coordenadas de un orden legítimo que le concede validez a la acción. El orden legítimo está expresado en las formas o instituciones políticas, sociales y económicas. También plantea Weber (1997, p.172), que la creencia en la legitimidad se fomenta con las relaciones sociales, y que esta no es un asunto logrado por unas cualidades individuales, es un efecto de las relaciones sociales donde las acciones están enmarcadas en unas máximas que presuponen unos ideales (deber ser).

Y, desde el funcionalismo, Durkheim en Las reglas del método sociológico (1986, p.38) cuando desarrolla la pregunta ¿qué es un hecho social? plantea la idea que todos los acontecimientos humanos son sociales, así introduce la noción de coacción y la presión que esta ejerce sobre los actores sociales con unas normas de conducta que son sociales. La influencia del exterior sobre el individuo tiene lugar por la corriente social, a diferencia de las creencias y prácticas constituidas, logra objetividad en la medida que es externa y tiene influencia sobre el individuo: "Así, en una asamblea, los grandes movimientos de entusiasmo, de indignación, de piedad que se producen, no tienen como lugar de origen ninguna conciencia particular" (Durkheim, 1986, p.42). 
Sería muy pretencioso definir qué es una institución en este trabajo, pero sí es posible plantear que existe coincidencia entre estos autores clásicos de la sociología que abordan el problema de la acción social. Con algunas rupturas con los sociólogos clásicos, pero conservando la idea de acción social y de actor en relación con la de sujeto se encuentra Touraine: "El actor, individual o colectivo, obra para hacer penetrar la racionalización y la subjetivación en una urdimbre de papeles sociales que tiende a organizarse según la lógica de la integración del sistema y del refuerzo del control” (Touraine, 2002, p.282). Estos autores coinciden en que existe un producto social que supera al individuo, que enmarca las acciones del individuo y le proporciona unos referentes o coordenadas para la actividad social, algo que no es el individuo pero se refleja en él y el individuo mismo es el garante de su existencia: las instituciones.

\section{La institucionalización de las prácticas}

Inicialmente es necesario establecer algunos acuerdos frente a lo que se entiende por institución, institucional e institucionalizado. Con lo planteado en el apartado anterior hay una idea global sobre la institución, la que "no es de ninguna manera limitada (solamente vuelta visible) por los muros del asilo o las fronteras de la asociación" (Lourau, 2001, p.28), la institución está directamente relacionada con las formas que resultan de la implicación del actor social en el sistema. Desde el institucionalismo económico, North plantea que: "Las instituciones son las reglas del juego en una sociedad o, más formalmente, son las limitaciones ideadas por el hombre que dan forma a la interacción humana. Por consiguiente, estructuran incentivos en el intercambio humano, sea político social o económico" (1993, p.13).

Ahora, lo institucional se refiera a lo que pertenece a una institución, a las acciones instauradas en las actividades de intercambio social ${ }^{6}$. Existen unas

6 Estas acciones instauradas para el intercambio social, son las que abren la posibilidad de enmarcar las prácticas y de darles la categoría de pertinencia y de pertenencia a un orden institucional. En el caso que se trata en el presente trabajo, la psicología educativa y las intervenciones psicoeducativas, su revisión desde el marco institucional es una vía para caracterizarlas y delimitar su campo de aplicación. 
pertenencias a las ordenaciones imaginarias instaladas en nuestra psique que operan en los diferentes procesos de intercambio social, económico o político:

La sociedad es creación, y creación de sí misma autocreación. Es la emergencia de una nueva forma ontológica - un nuevo eidos- y de un nuevo nivel y modo de ser. Es una cuasi totalidad cohesionada por las instituciones (lenguaje, normas, familia, modos de producción) y por las significaciones que estas instituciones encarnan (tótems, tabúes, dioses, Dios, polis, mercancía, riqueza, patria, etc.). Ambas-instituciones y significaciones- representan creaciones ontológicas. (Castoriadis, 1997, p.4)

Esas significaciones que encarnan las instituciones no son solo costreñidoras, también operan como posibilitadoras de la acción. Es importante señalar que desde otras perspectivas se han incluido las categorías de agente y de agencia para aludir al actor social y a su acción, "Dondequiera que es necesario tomar decisiones de acción, las instituciones pueden indicarle al agente el camino y, a menudo, incluso se hacen cargo de la decisión" (Wieland, 1996, p.42).

Por su parte, lo institucionalizado, se refiere al conjunto de relaciones establecidas entre el actor y el sistema. Debe diferenciarse lo instituido y lo instituyente: Lo instituido es lo que ejerce dominación y, la pasividad frente a ello es lo instituyente. Existe una racionalidad instituida en las acciones sociales desarrolladas por los actores, esa racionalidad instituida tiende a ser instituyente, la pasividad frente a ella la hará instituyente. En términos de la práctica psicológica se ve así:

Si hay una perspectiva aplicacionista de la psicología, está dada por la racionalidad instituida del funcionamiento como ciencia básica, que conlleva a la pasividad del fenómeno abordado porque solo le interesa comprenderlo 
y teorizar sobre él; esto, a veces se asume como intervención. Pero la posibilidad transformadora de la ciencia por aplicar, de la tecnología sobre el fenómeno permite la ruptura del ciclo instituido-instituyente que reproduce y tiende a perpetuar el orden social como es. De la dinámica de la presión de lo instituido sobre el fenómeno social que no es pasivo, si el papel de la ciencia aplicada se lo permite, se podrá presentar la emergencia de un producto social diferente, por supuesto establecido en relación con los marcos institucionales ${ }^{7}$.

En algunas intervenciones psicoeducativas se puede ver la emergencia de nuevos productos sociales en los diferentes marcos institucionales: Levy, Hasson, Kravetz y Roe (2012) desarrollaron intervenciones psicoeducativas con las familias en el marco de la salud mental para apoyar el trabajo de los padres en la rehabilitación psiquiátrica de los hijos, y plantean que el aporte de la psicoeducación está de lado de las habilidades de comunicación y del aporte de información. Espín (2009) presenta la evaluación favorable del uso de un programa psicoeducativo para cuidadores de adultos mayores con demencia, en él aplicaron intervenciones psicoeducativas con los cuidadores para aumentar su calidad de vida ligado a grupos de educación e información combinados con asesoramiento individual. De igual forma Cervantes, Espín, Román, Sánchez, Ibáñez, Martín y Palao (2013) evaluaron el impacto del trabajo psicoeducativo en 72 pacientes con diabetes tipo 2 , al que se refieren como una intervención educativa sobre las conductas de los pacientes diabéticos (p.500). Casañas, Catalán, Raya y Real (2014) evaluaron la efectividad de una intervención psicoeducativa, que está dirigida a pacientes con síntomas depresivos en atención primaria; encontraron que era más eficaz en los pacientes con síntomas leves, además agregan que la pueden desarrollar enfermeras (p.146).

Farkas, Hernández y Santelices (2010) investigan sobre los momentos de

7 Esto no quiere decir que el producido sea siempre algo esperado, puede ser lo inesperado y posiblemente hasta lo indeseado socialmente. 
cambio en mujeres embarazadas primigestantes, presentados en un proceso terapéutico en talleres grupales psicoeducativos, entre varios hallazgos presentan que los indicadores de momentos de cambio se comportan diferente en intervenciones psicoeducativas que en intervenciones psicoterapéuticas, por lo que sugieren mayor investigación (pp.420-421). Martínez, Albein, Múnera y Verdejo (2012) también evaluaron el impacto de la psicoeducación en los familiares para acompañar el tratamiento de pacientes dependientes del alcohol, la psicoeducación en este caso también tiene un fuerte componente informativo que permita a la familia interpretar "las conductas molestas en términos sintomáticos” (p.19). Todos estos trabajos están en el marco de la salud. Por último, los autores Dionne \& Altamirano (2012) en "Los desafíos de un verdadero sistema de justicia juvenil: una visión psicoeducativa" plantearon "una perspectiva psicoeducativa, como en el presente trabajo, centrada en el joven como persona, en su familia y en su entorno social" (p.1056), un trabajo más centrado en el aspecto social.

Los ejemplos de intervenciones psicoeducativas son abundantes en la literatura especializada de diferentes áreas: Salud, educación, deporte y trabajo con comunidad entre otras y es común ver que tienen un componente fuerte en lo referido a propiciar espacios para la información sobre las problemáticas y a desarrollar habilidades para el control y manejo de situaciones específicas. En las intervenciones psicoeducativas se propicia una situación enseñanza-aprendizaje, a lo que se suma que son una técnica y que su aplicación no es exclusivamente de los psicólogos. Los marcos institucionales acunan estas prácticas institucionalizadas que funcionan como correlatos de los procesos de socialización fruto de una actividad humana y de un ordenamiento social que "Tanto por su génesis (el orden social es resultado de la actividad humana pasada), como por su existencia en cualquier momento del tiempo (el orden social solo existe en tanto que la actividad humana siga produciéndolo), es un producto humano" (Berger \& Luckmann, 2001, p.73) con las variaciones que ello implica. 
Las diferentes instituciones ${ }^{8}$ como productos estrictamente humanos, operan como ordenadoras de significaciones: La escuela es un ejemplo de una institución -de socialización primaria- en la que se ordenan las significaciones sobre la excelencia. "De este modo la escuela no es el lugar en el que se producen las desigualdades sociales sino donde se legitiman esas desigualdades" (Álvarez \& Varela, 2009, p.62) a continuación un ejemplo que pone en escena la escuela y la psicología:

Los excluidos son condenados en nombre de un criterio colectivamente reconocido y aprobado, por lo tanto psicológicamente indiscutible e indiscutido, el de la inteligencia: con lo que a menudo no les queda otro remedio para restaurar una identidad amenazada que las rupturas brutales con el orden escolar y el orden social (se ha observado en Francia que en la rebelión contra la escuela es donde se fraguan y se moldean muchas bandas de delincuentes) o, como también es el caso, la crisis psíquica, incluso la enfermedad mental o el suicidio. (Bourdieu, 1997, p.44)

Con estos insumos, se trata de establecer la relevancia del proceso de institucionalización de la práctica psicológica y más aún la importancia de prevalecer el interés en la intención de mantener las instituciones en una época en la que se habla de la crisis de las instituciones". En el "fenómeno de desmodernización que conlleva la ruptura del actor con el sistema, se presenta la desinstitucionalización y la desocialización" (Touraine, 2012, p.45). Por su parte, la psicología también asiste en cada uno de sus ámbitos a este proceso y a la desaparición de roles que conlleva la desocialización.

8 Álvarez y Varela (2009) presentan las instituciones en varias categorías: Instituciones de socialización primaria, instituciones de resocialización, e instituciones de socialización secundaria.

9 Touraine (2012, p.47) propone una vía de análisis que parte de la idea de desmodernización caracterizada por la desocialización y la desinstitucionalización en la que rescata la importancia del viraje del interés hacia el actor. 


\section{Psicología de la educación e intervenciones psicoeducativas como prác- ticas institucionalizadas}

Una práctica institucionalizada es "una práctica sedimentada a la mayor profundidad en un espacio tiempo" (Giddens, 1995, p.58), esto introduce dos elementos concretos y fundamentales, espacio y tiempo, a tener en cuenta para hablar de la práctica psicológica como práctica institucionalizada en la que el agente, el psicólogo, además de las cualidades psicológicas, se le suman las propias del rol social como profesional.

En el caso de la psicología los contextos de interacción varían según el ámbito o el campo de aplicación y es fundamental la postura del actor social, del psicólogo, para el desarrollo de las tareas. Cuando se asume la posición aplicacionista la psicología y la educación tienen que ver con todo, se hacen funcionar como un método que se aplica a todo, pero: ¿son la psicología y la educación métodos? Si se entiende por método "Modo ordenado de proceder para llegar a un resultado o fin determinado, especialmente para descubrir la verdad y sistematizar los conocimientos" (Ferrater, 2004, p.2400) la respuesta es negativa, la psicología y la educación no son métodos. Pero, la educación es una práctica social y la psicología es considerada como una ciencia o una disciplina. $\mathrm{Y}$ ambas se valen de diferentes métodos en sus procedimientos.

En lo referente a la educación, en el caso de la Ley 115 de 1994, la Ley General de la Educación colombiana, el artículo $1^{\circ}$ define la educación como "un proceso de formación permanente, personal, cultural y social que se fundamenta en una concepción integral de la persona humana, de su dignidad, sus derechos y sus deberes". A esta definición le subyace la concepción de la educación como una práctica fundada en relaciones sociales. Y la psicología educativa -como psicología aplicada- es considera una tecnología que le interesa intervenir sobre las prácticas educativas, sobre las prácticas sociales de educación (donde se pueden incluir las intervenciones psicoeducativas). Esto enmarca la educación, la psicología educativa y las intervenciones psicoeducativas en el terreno de las relaciones sociales que son 
el espacio para las acciones sociales ${ }^{10}$, y ellas son las que generan el espacio para el sentido.

La educación obedece a un esquema de racionalidad: lo racional que se materializa en un compendio de reglas que va a garantizar el cuidado del niño, su domesticación y desarrollo. "Esas reglas y su racionalidad son de propiedad de instancias como los educadores y como los médicos, como el saber pedagógico, como el saber médico" (Foucault, 2001, pp.241-242). En la conjunción de las acciones sociales de varios individuos se establece la relación social:

Todo se cumple dentro de un sistema institucional, y es con esta condición que el hombre, pese a no estar obligado en absoluto por ninguna institución (en el sentido en que estaría obligado por la ley) está referido a todo el sistema, o puede inscribirse en múltiples posiciones dentro del sistema. (Lourau, 2001, p.67)

En este orden de ideas, se pueden observar las instituciones educativas ${ }^{11}$ como contenedor y posibilitador de la existencia de la psicología educativa en la medida en que en ellas tiene origen su objeto que es lo que soporta y justifica la existencia de cualquier técnica, inclusive de la psicología y las instituciones de salud como contenedoras y posibilitadoras de la psicología clínica, así se puede pensar sobre los diferentes campos aplicados de la psicología. En cada contexto institucional funciona un orden legítimo que concede validez a cada acción, "La participación en una entidad social implica un compromiso y al mismo tiempo una adhesión" (Goffman, 1961, p.175). Hacer intervenciones psicoeducativas desde la perspectiva aplicacionista $^{12}$, hace que funcione como una gramática que proporciona los límites y las posibilidades de movimiento e implica un gran riesgo:

10 Categoría que incluye las prácticas educativas y las prácticas psicológicas.

11 Que no se entienda por institución educativa solo la escuela.

12 Esto es teniendo la psicología educativa como ciencia básica y asumir la posición de saberlo todo desde la psicología. 
Pero cuando una gramática o un esquema de pensamiento están ya constituidos, cualquier cosa que se produzca en su interior da una sensación de "ya dicho", de "ya pensado", una sensación de que pisamos terreno conocido, de que podemos seguir hablando o pensando en su interior sin dificultades, sin sobresaltos, sin sorpresas. (Larrosa, 2006, p.2)

Y no se genera la posibilidad de movilización teórica y técnica y se continúa la intervención desde categorías sedimentadas y técnicas consolidadas que ubican al psicólogo y la psicología en el lugar de la verdad. Y en esa tendencia, con esa verdad, se aborda cualquier problema sin importar su contexto y los psicólogos intervienen en cualquier ámbito o en el terreno de otros saberes, con los mismos conceptos, las mismas categorías y las mismas técnicas:

Entre esas variables habría que destacar el tema de la superpoblación de psicólogos y el preocupante tema del intrusismo profesional, el cual puede ser practicado desde otras profesiones afines o también por los mismos psicólogos que estando trabajando en un área determinada empiezan a trabajar en otra área sin la especialización adecuada. Es este último caso más grave, ya que son los propios psicólogos los que colaboran en crear una imagen pobre de nuestra profesión. (Ardila, 2002, p.183)

Desde lo propuesto se trata de dar un orden a las intervenciones psicoeducativas desde su institucionalización para definir el ámbito en el que se circunscriben y entenderlas como una técnica derivada de la psicología educativa que ha sido adoptada por otros ámbitos y otros saberes. De esta suerte el que hace intervenciones psicoeducativas no hace necesariamente psicología educativa, puede estar haciendo psicología clínica, de la salud, organizacional, deportiva o... puede, inclusive, no ser psicólogo. 


\section{Discusión y Conclusiones}

En la conjunción de las acciones sociales de varios individuos se establece la relación social. Los participantes de la relación social orientan su acción hacia los otros en las coordenadas de un orden legítimo que le concede validez a la acción. La posición del psicólogo puede encontrar alguna distancia de la psicología como tal, hay psicólogos y hay psicología. La psicología está en el orden institucional del discurso, los(as) psicólogos(as) en esta época de la técnica pueden quedar reducidos y reducir a los otros, a la categoría de recursos, por lo tanto mantener en su foco las fuerzas institucionales puede poner límites a la técnica y ver que allí en la institución, donde reside el peligro, también reside la opción.

- La psicología logra su pertinencia en la sociedad, en los marcos institucionales, a través de los diferentes ámbitos de aplicación entre los que está la psicología educativa. De igual forma, de los diferentes ámbitos se derivan técnicas que también logran su pertinencia dentro los contextos específicos donde se aplican. Las psicoeducación y las intervenciones psicoeducativas son técnicas de intervención que se usan en diferentes contextos que las contienen como prácticas sociales y las definen en referencia al ámbito específico del contexto.

- De esta forma es posible hacer intervenciones psicoeducativas y psicoeducación por fuera de los contextos escolares y educativos y por fuera del ámbito de la psicología educativa. Sin embargo están en referencia con el orden legítimo que está expresado en las formas o instituciones políticas, sociales y económicas y desde ellas se define su pertenencia a un ámbito específico que no siempre será la psicología educativa o la psicología en general.

- Definir, por defecto, que cualquier intervención psicoeducativa o de psicoeducación pertenece al ámbito de la psicología educativa es usarla como una verdad de la que deriva un ejercicio de poder en el que se supone la universalidad y la intemporalidad de la práctica, es desconocer el contexto y la institucionalización de la práctica. Hoy las relaciones se plantean de otra forma y la psicología educativa busca su identidad 
en medio del agotamiento de los modelos derivados de los criterios de normalidad venidos de los modelos médicos normalizadores.

- Las prácticas psicológicas, como prácticas discursivas, son desarrolladas por los psicólogos en los contextos sociales que se enmarcan en los órdenes institucionales y en ellos se legitiman. Por eso no hay una manera esencial de hacer psicología; las prácticas psicológicas son producto de la cultura, la historia y los discursos. Funcionan como gramáticas finitas con posibilidades de producción infinita. Las instituciones ocupan, frente a la práctica psicológica, el mismo lugar que frente a las otras técnicas que funcionan en ellas: Las instituciones no ocupan un lugar en la práctica del psicólogo: la práctica del psicólogo tiene su lugar en lo institucional.

\section{Referencias}

Alarcón, M. \& Vargas, M. (2012). Evaluación de competencias psicoeducativas a partir de un programa de formación especializada para profesionales de intervención directa con adolescentes infractores y no infractores. Universitas Psychologica, 4(11). Disponible en: http:// revistas.javeriana.edu.co/index.php/revPsycho/article/view/1115

Althusser, L. (1976). Ideología y aparatos ideológicos de Estado. Medellín: Editorial la Oveja Negra.

Álvarez, F. \& Varela, J. (2009). Sociología de las instituciones. Bases sociales y culturales de la conducta. Madrid: Ediciones Morata, S. L.

Ardila, R. (2002). Entrevista a Francisco Santolaya. En La psicología en el futuro (pp.180-183). Madrid: Ediciones Pirámide.

Ardila, R. (2011). El mundo de la psicología. Bogotá: Editorial Manual Moderno.

Beltrán, J. \& Bueno, J. (1995). Psicología de la educación. Barcelona: Editorial Boixareu Universitaria.

Berger, P. \& Luckmann, T. (2001). La construcción social de la realidad. Buenos Aires: Amorrortu.

Bourdieu, P. (1997). Razones prácticas. Barcelona: Anagrama. 
Brugger, W. (1995). Diccionario de Filosofía. Barcelona: Editorial Herder. Cabrera, C., Ruiz, L., González, G., Vega, M. \& Valadez, I. (2009). Efecto de una intervención psicoeducativa para disminuir el Síndrome Burnout en personal de confianza de la Comisión Federal de Electricidad. Salud Mental, 31, 215-221. Disponible en: http://www.redalyc. org/articulo.oa?id=58212279005

Casañas, R., Catalán, R., Raya, A. \& Real, J. (2014). Efectividad de un programa grupal psicoeducativo para la depresión mayor en atención primaria: ensayo clínico controlado aleatorizado. (Spanish). Revista de la Asociación Española de Neuropsiquiatría, 34(121), 145-146. doi: 10.4321/S0211-57352014000100009.

Castejón, J., González, C., Gilar, R. \& Miñano, P. (2010). Psicología de la educación. San Vicente, Alicante, España: Editorial Club Universitario.

Castoriadis, C. (1997). El Imaginario Social Instituyente. (versión online). Zona Erógena, 35. Disponible en: http://www.ubiobio.cl/miweb/webfile/media/267/Castoriadis\%20Cornelius\%20-\%20El\%20Imaginario\%20Social\%20Instituyente.pdf

Cervantes, M.A., Espín, N.G., Román, J.B., Sánchez, M.N., Ibáñez, P.B., Martín, P.V. \& Palao, J.S. (2013). Psychoeducative groups help control type 2 diabetes in a primary care setting. Nutricion Hospitalaria, 28(2), 497-505. doi:10.3305/nh.2013.28.2.6063.

Coll, C. (1983). Psicología social de la educación: ciencia, tecnología y actividad técnico-práctica. Estudios de Psicología, 14-15, 168-193. Disponible en: http://dialnet.unirioja.es/servlet/articulo?codigo=65890

Coll, C. (Ed.) (1997). Desarrollo psicológico y educación. Madrid: Alianza Editorial.

Coll, C. (Ed.) (1998). Psicología de la educación. Cataluña, España: Ediciones de la Universidad Oberta de Cataluña.

Coll, C. (2004a). La misión de la escuela y su articulación con otros escenarios educativos. Reflexiones en torno al protagonismo y los límites de la educación escolar. En COMIE (Ed.), VI Congreso Nacional de 
Investigación Educativa. Conferencias magistrales (pp.15-56). México, DF: Comité Mexicano de Investigación.

Coll, C. (2004b). Psicología de la educación y prácticas educativas mediadas por las tecnologías de la información y la comunicación. Una mirada constructivista. Revista Electrónica Sinéctica, 25, 1-24.

Coll, C. \& Monereo, C. (Eds.) (2008). Psicología de la educación virtual. Aprender y enseñar con las tecnologías de la educación y la información. Madrid: Ediciones Morata.

Dionne, J. \& Altamirano, C. (2012). Los desafíos de un verdadero sistema de justicia juvenil: una visión psicoeducativa. (Spanish). Universitas Psychologica, 11(4), 1055-1064.

Durkheim, E. (1986). Las reglas del método sociológico. México: Fondo de Cultura Económica.

Espín, A. (2009). Escuela de Cuidadores como programa psicoeducativo para cuidadores informales de adultos mayores con demencia. Revista Cubana Salud Pública [online], 2(35). Disponible en: http://scielo.sld. cu/scielo.php?script=sci_arttext\&pid=S0864-34662009000200019

Farkas, Ch., Hernández, B. \& Santelices, M. (2010). Análisis de momentos de cambio en el proceso terapéutico adelantado bajo la modalidad de un taller grupal psicoeducativo con embarazadas primigestantes. [online]. Universitas Psychologica, (9). Disponible en http://www. scielo.org.co/pdf/rups/v9n2/v9n2a09

Fernández, B. (2011). Evolución y desarrollo de los modelos de intervención psicoeducativa en España. Psicología Educativa, 1(17), 27-37.

Ferrater, J. (2004). Diccionario de Filosofía. Barcelona: Ariel.

Foucault, M. (1983). La verdad y las formas jurídicas. México: Gedisa.

Foucault, M. (2001). Los anormales. México: Fondo de Cultura Económica. Foucault, M. (2005). El orden del discurso. Barcelona: Tusquets Editores. Galve, J. (2009). Intervención psicoeducativa en el aula con TDAH. Psicología Educativa, 2(15), 87-106. doi:10.5093/ed2009v15n2a2.

Giddens, A. (1995). La constitución de la sociedad. Bases para la teoría de la estructuración. Buenos Aires: Amorrortu. 
Goffman, E. (1961). Internados. Ensayos sobre la situación social de los enfermos mentales. Buenos Aires: Amorrortu.

Hernández, O. (2012). La psicología educativa en la escuela y la escuela en la psicología educativa. Un análisis del caso colombiano. En G. Marciales (Ed.), Psicología educativa: trayectorias, convergencias y vórtices. Bogotá: Editorial Universidad Javeriana.

Larrosa, J. (2006). Algunas notas sobre la experiencia y sus lenguajes. Serie encuentros y seminarios. Valladolid: Instituto Superior de Filosofía. Disponible en: http://www.me.gov.ar/curriform/publica/ oei_20031128/ponencia_larrosa.pdf

Levy-Frank, I., Hasson-Ohayon, I., Kravetz, S. \& Roe, D. (2012). A Narrative evaluation of a psychoeducation and a therapeutic alliance intervention for parents of persons with a severe mental illness. Family Process, 51(2), 265-280. doi:10.1111/j.1545-5300.2012.01398.x.

Lourau, R. (2001). El análisis institucional. Buenos Aires: Amorrortu.

Lourau, R. (2001). Libertad de movimientos. Una introducción al análisis institucional. Buenos Aires: Eudeba.

Martínez, J., Albein, N., Munera, P. \& Verdejo, A. (2012). La psicoeducación como estrategia para mejorar el apoyo familiar percibido del paciente con dependencia al alcohol y trastorno de la personalidad. International Journal of Psychological Research, 5(1), 18-24.

Marx, C. (1959). El capital. Crítica de la economía política. (2a ed.). México: Fondo de Cultura Económica.

Marx, C. (1978). El dieciocho brumario de Luis Bonaparte. República Popular de China: Ediciones en Lenguas Extranjeras.

Navas, C., Villegas, H., Hurtado, R. \& Zapata, E. (2007). Programa de intervención psicoeducativo para el apoyo del paciente con cáncer. Rev. Venezolana de Oncología, 1(19), 84-93.

North, D. (1993). Instituciones, cambio institucional y desempeño económico. México, D.F.: Fondo de Cultura Económica.

Parsons, T. (1961). Theories of society. New York: The Free Press of Glencoe. 
República de Colombia (1994). Ley 115 de 1994. Ley General de la Educación. Diario Oficial No. 41.214 del 8 de febrero de 1994.

Rico, M. (1997). Investigación en trastornos del desarrollo. En M. Moreno (Ed.), Intervención psicoeducativa en las dificultades del desarrollo. Barcelona: Ariel Educación.

Rigo, M., Díaz, F. \& Hernández, G. (2005). La psicología de la educación como disciplina y profesión. (Entrevista con César Coll). Revista Electrónica de Investigación Educativa, 1(7). Disponible en: http:// redie.uabc.mx/vol7nol/contenido-rigo.html

Touraine, A. (2002). Crítica de la modernidad. (2a ed.). México, D.F.: Fondo de Cultura Económica.

Touraine, A. (2012). ¿Podremos vivir juntos? (2a ed.). México, D.F.: Fondo de Cultura Económica.

Weber, M. (1997). Economía y sociedad. (Vol. 1). Santafé de Bogotá: Fondo de Cultura Económica.

Wieland, W. (1996). La razón y su praxis. Cuatro ensayos filosóficos. Buenos Aires: Editorial Biblos. 